\title{
UN ANÁLISIS DEL MECANISMO DE LA CONFORMIDAD ${ }^{1}$
}

An analysis of plea bargaining mechanism

\author{
ALICIA MONTERO MOLERA² \\ Universidad de Castilla-La Mancha
}

\begin{abstract}
RESUMEN
El mecanismo de la conformidad (o plea bargaining) se ha convertido en la actualidad en la forma de resolución de conflictos penales más habitual en la mayoría de los países. Según demuestran algunas investigaciones, la negociación propia del acuerdo de conformidad entre la defensa y la acusación carece de transparencia y cuenta con una supervisión judicial mínima. Amparándose en el principio de economía procesal, los procesos de conformidad son ágiles y sencillos, evitando la celebración del juicio y obteniendo el acusado aparentemente una rebaja de la pena en la condena impuesta por el juez. Sin embargo, se cuestiona la voluntariedad real del imputado que decide conformarse y se abre la puerta a la posible condena de personas inocentes que prefieren asumir una pena leve que enfrentarse al riesgo de una pena mayor en juicio. La importancia de la percepción del paso por la justicia de aquellos que se conformen y las posibles consecuencias prácticas que tendrá la conformidad en las personas condenadas se discuten en este estudio.
\end{abstract}

\section{PALABRAS CLAVE}

Conformidad, toma de decisiones, justicia negociada.

\section{ABSTRACT}

Nowadays most of criminal convictions come from negotiated pleas in most of the countries. According to some investigations, the negotiation between prosecution and the lawyer is characterized by its lack of transparency and it has minimal judicial supervision. Based on the procedural agility, plea decisions are made quickly, defendants avoid the trial and they apparently obtain a reduction in charges or sentence imposed by the judge. However, the real voluntariness of the defendant who decides accepting a deal is questioned. There are many reasons to believe that there is a high number of false guilty pleas, people who prefer to assume a light sentence than face the risk of a greater sentence at trial. The importance of integrating procedural justice norms into plea bargaining is discussed in this study.

\section{KEYWORDS}

Plea bargaining, sentencing, negotiated justice.

\section{Introducción}

Generalmente, los regímenes jurídicos contemplan que un procedimiento penal finalice con una sentencia tras la celebración de un juicio, sin embargo, en este último siglo, multitud de países han previsto un modo de terminación del procedimiento alternativo a la celebración del juicio, alcanzando un acuerdo de conformidad.

\footnotetext{
${ }^{1}$ Este trabajo ha sido elaborado en el marco del proyecto "Justicia penal para todos. Un estudio del funcionamiento y la accesibilidad de la justicia penal" (Ref. SBPLY/17/180501/00027), que ha sido financiado por la Consejería de Educación, Cultura y Deportes de la Junta de Comunidades de Castilla-La Mancha y cofinanciado por el Fondo Europeo de desarrollo regional.

${ }^{2}$ Investigadora contratada en el Centro de Investigación en Criminología de Castilla-La Mancha (Albacete, España). Correspondencia: alicia.montero@uclm.es.
} 
La conformidad ha sido introducida como un mecanismo de justicia negociada en diversos ordenamientos de nuestro entorno con el objetivo de gestionar con menor esfuerzo procesal y de recursos un volumen importante de casos $^{3}$.

Estos acuerdos implican una terminación temprana del proceso y acostumbran a ser el producto de una negociación entre los profesionales de la acusación y la defensa (en la mayor parte de los casos, fiscal y abogado). Estas negociaciones suelen tener lugar en espacios informales y de escasa visibilidad, aunque posteriormente el acuerdo se presentará ante el juez ${ }^{4}$.

La naturaleza del mecanismo de la conformidad no resulta una cuestión demasiado controvertida, ya que la doctrina se ha posicionado mayoritariamente entendiendo que existe una semejanza entre esta institución y el allanamiento propio del proceso civil realizado en el ejercicio del principio de oportunidad ${ }^{5}$.

De este modo, la conformidad consiste en un "acto por el que tanto el acusado como su abogado aceptan, bajo ciertos límites, la pena solicitada por el Ministerio Fiscal y la acusación particular, o la más grave de las interesadas, y, previo control por parte del Juez de los términos del acuerdo, este dicta sentencia inmediata sin necesidad de celebrarse la vista" ${ }^{\prime \prime}$. Desde otro punto de vista, se entiende la conformidad como "un acto de disposición del acusado sobre su inocencia, consistente en renunciar a su presunción y, por tanto, a su defensa, y en aceptar su participación en los hechos, así como las consecuencias jurídicas pedidas por el acusador"7.

En este proceso de toma de decisiones, los fiscales deben decidir si quieren ofrecer un trato de conformidad y definir la pena y su duración, los abogados deben informarse adecuadamente acerca de los extremos del acuerdo para decidir cómo asesorar al cliente adecuadamente, los acusados deben decidir si aceptar este trato de conformidad o arriesgarse a ir a juicio, y los jueces deben decidir si aceptar los términos alcanzados con la conformidad.

Este ha sido un campo poco explorado desde el punto de vista penal y criminológico hasta hace pocos años ${ }^{8}$, sin embargo, debido a su alta prevalencia en los sistemas de justicia actuales, la institución de la conformidad está recibiendo gran atención por parte de la comunidad científica internacional.

Así, en Estados Unidos, país originario de este mecanismo, en los últimos años, alrededor del $90-95 \%$ de las condenas se obtienen mediante una asunción de culpabilidad del acusado, o también conocidos como guilty plea $^{9}$, siendo la mayoría de ellas fruto de haber alcanzado un acuerdo de conformidad ${ }^{10}$.

Esta expansión generalizada del uso de la conformidad como forma de finalización del proceso penal se puede observar también en una encuesta reciente realizada en noventa países de los cinco continentes, que reveló que el $72 \%$ de los países contemplan el mecanismo de la conformidad en sus sistemas judiciales. Asimismo, se ha observado un incremento en el número de acuerdos por conformidad en los últimos años en países diversos como China, Rusia, Sudáfrica o Georgia ${ }^{11}$.

Por último, la investigación empírica ha demostrado que la forma en la que se atienda a las exigencias procedimentales durante el proceso de la conformidad y la percepción que tenga la persona imputada por su paso por la justicia conlleva grandes implicaciones en términos de justicia procedimental que deben ser examinadas.

\footnotetext{
${ }^{3}$ LANGER (2004), pp. 39-45.

${ }^{4}$ REDLICH et al. (2017), p. 8.

${ }^{5}$ FRAGA (2016), p. 15.

${ }^{6}$ BARONA (1994), p. 56.

7 ZARZALEJOS (2011), p. 4.

${ }^{8}$ DEL MORAL (2002), p. 1.

${ }^{9}$ DEVERS (2011), p. 1; VIANO (2012), p. 118.

${ }^{10}$ Este hecho ha llevado a admitir a la Corte Suprema de Estados Unidos que su sistema de justicia es "un sistema de conformidades,

no un sistema de juicios" (Lafter v. Cooper, 2012).

11 FAIR TRIAL INTERNATIONAL (2016), pp. 22-23.
} 


\section{Origen del plea bargaining}

El plea bargaining ${ }^{12}$ (sistema de conformidades) constituyó el origen de la justicia penal negociada, un sistema que se popularizó en Estados Unidos durante el siglo XIX, hasta convertirse en la forma habitual de resolver las cuestiones penales de este país ${ }^{13}$.

Antes de la aparición del plea bargaining, los juicios eran rápidos y baratos por lo que las conformidades eran innecesarias. Con la aparición de los letrados en los procedimientos penales, el proceso se volvió mucho más complejo y los tribunales y fiscales comenzaron a buscar formas de alentar las declaraciones de culpabilidad ${ }^{14}$.

Los autores estadounidenses no mantienen una posición unánime a la hora de valorar la naturaleza del plea bargaining; ha sido calificado como un compromiso ${ }^{15}$, como un contrato ${ }^{16} \mathrm{o}$ como un desastre ${ }^{17}$.

La esencia de la problemática de la conformidad reside en la tensión constante entre el principio de legalidad y el de oportunidad, el cual se halla estrechamente ligado a esta institución. En los países de la common law, donde rige el modelo acusatorio y el principio de oportunidad, los fiscales tienen la facultad de abstenerse de acusar cuando carecen de pruebas suficientes o por otras razones denominadas "intereses públicos" justificando tal abstención ${ }^{18}$. En contraposición se encuentra el modelo inquisitivo y el principio de legalidad, principio que rige en la mayoría de los países continentales europeos y que obliga a los fiscales a acusar cuando existe suficiente evidencia de culpabilidad.

En este contexto se ubica la conformidad, una manifestación del principio de oportunidad reglada, donde se admite la facultad de negociación para determinados delitos, respetando determinados límites y los principios de legalidad penal y procesal ${ }^{19}$.

Hay varios aspectos del sistema de justicia penal estadounidense que favorecen el sistema de conformidades en este país. En primer lugar, el carácter adversarial de su procedimiento judicial coloca al juez en una situación pasiva, como un "árbitro", permitiendo que las partes lleven la iniciativa durante el proceso penal. El juez no puede tomar ninguna medida para hallar, verificar o evaluar de manera independiente la información sobre el caso a fin de evaluar la evidencia en contra del acusado. Así, fiscalía y defensa controlan claramente el resultado del caso $^{20}$.

En segundo lugar, la discrecionalidad sustancial de los fiscales les concede el poder legal y la flexibilidad para negociar, especialmente cuando las probabilidades de obtener una condena en juicio no son muy altas o cuando hay multitud de $\operatorname{casos}^{21}$. En este sentido, los fiscales motivados para conseguir una percepción pública favorable utilizarán el plea bargaining con el fin de maximizar su número de condenas, así, al resolver los casos mediante conformidades, el fiscal evita el riesgo de absolución y ahorra recursos que pueden utilizarse en otros $\operatorname{casos}^{22}$. Los fiscales están dispuestos a aceptar una sentencia más benévola cuando se asegura la probabilidad de condena y los costes son menores que los de un juicio.

\footnotetext{
${ }^{12}$ En Estados Unidos, las Federal Rules of Criminal Procedure (en concreto, la regla 11) determinan los principios y exigencias a las que debe someterse el guilty plea. Además, por un lado, los fiscales cuentan con una guía (la Attorneys Manual) que actúa como protocolo de actuación en cuanto a los diferentes escenarios que puede plantear el plea bargaining; y, por otro lado, los jueces cuentan con una guía recopilatoria (Benchbook for U.S District Court Judges) donde se recogen recomendaciones y propuestas para actuar correctamente en el plea collloquy.

${ }^{13}$ FERRÉ (2018), p. 2.

${ }^{14}$ ALSCHULER (1979), pp. 1-6.

${ }^{15}$ EASTERBROOK (1983), p. 1969.

${ }^{16}$ SCOTT Y STUNTZ (1992), p. 1909

17 SCHULHOFER (1992), p. 2004; FERRÉ (2018), p. 16.

${ }^{18}$ GAZAL-AYAL Y RIZA (2009), pp. 3-4.

${ }^{19}$ FERRÉ (2018), p. 2.

${ }^{20}$ VIANO (2012), p. 111.

${ }^{21}$ VIANO (2012), p. 111

22 LANDES (1971), pp. 62-66; SCHULHOFER (1988), p. 46.
} 


\section{El proceso de toma de decisión de la conformidad}

Con una clara inspiración proveniente del instituto del plea bargaining norteamericano, muchas legislaciones europeas y latinoamericanas han incorporado en sus sistemas procesales figuras similares dentro de la justicia penal negociada. En este sentido, se encuentra el patteggiamiento italiano, la Absprache alemana o la conformidad española, entre otros ${ }^{23}$.El proceso seguido para adoptar una sentencia de conformidad reúne algunas características comunes aplicables a la mayoría de los países que contemplan este mecanismo, sin embargo, debe señalarse que la vigencia del principio de legalidad en el ámbito jurídico europeo y sudamericano no permite que se disponga de forma tan amplia de la acción penal, de modo que algunos tipos de acuerdos como el change bargaining (permite negociar los hechos) o lateral bargaining (permite negociar la calificación) no pueden adoptarse ${ }^{24}$.

En la negociación de los extremos que abarca el acuerdo de conformidad, el fiscal y el abogado defensor pueden negociar sobre cualquier elemento del caso, siendo objeto los elementos más comunes de esta negociación la severidad de los cargos por los que acusa el fiscal, los elementos de hecho y la duración de la pena. De esta forma, se pueden ver alterados los hechos fácticos del delito, obviando y ocultando algunos detalles (por ejemplo, el uso de un arma) que reducirán los cargos, las consecuencias colaterales y, en definitiva, la sentencia ${ }^{25}$.

Después de que tenga lugar esa negociación entra la acusación y la defensa, se celebrará una audiencia en la que el juez revisará e informará al acusado sobre los términos acordados en el acuerdo, asegurando que el acusado entiende plenamente su alcance. Existe poca información recogida de forma sistemática sobre lo que los jueces suelen preguntar al imputado durante la audiencia de la conformidad, pero tiende a describirse este proceso como una formalidad "repetitiva" que hace poco para informar o proteger al acusado ${ }^{26}$ habiéndose constatado que el contenido de la información suministrada por los juzgados a los acusados es muy variable ${ }^{27}$.

La decisión por la que un acusado se declara culpable y acepta un acuerdo de conformidad debe ser constitucionalmente válida. En este sentido, el juez es el encargado de asegurar que la decisión es adoptada de manera decisión debe consciente, inteligente y voluntaria y que cuenta con una base fáctica adecuada ${ }^{28}$. Así, Además, con el fin de que el tribunal pueda reconocer y evaluar la declaración de culpabilidad, la Regla Federal 11 (b) (3) requiere que antes de emitir un fallo basado en la guilty plea, el tribunal debe examinar si existe una base fáctica sobre la que apoyarse, si la decisión es voluntaria, y si está plenamente informado de los derechos que posee y de los cargos y pena que se le puede imponer ${ }^{29}$.

La primera característica de una declaración de culpabilidad reside en que el acusado está dispuesto a aceptar la responsabilidad penal por el delito cometido ${ }^{30}$. La decisión de aceptar un acuerdo de conformidad debe ser adoptada de manera consciente, conociendo el acusado la naturaleza de los cargos, las consecuencias que derivan de la naturaleza de la sentencia y los derechos a los que está renunciando ${ }^{31}$.

El requisito de la consciencia alcanza también a la renuncia de ciertos derechos constitucionales, ya que cuando el acusado se declara culpable y alcanza un acuerdo de conformidad no solo acepta todos los efectos derivados de una sentencia condenatoria sino que también renuncia al derecho a un juicio justo, a la presunción de inocencia, a elegir una

${ }^{23}$ NIEVA (2019), p. 327.

${ }^{24}$ FRAGA (2018), pp. 56-57.

25 PIZZI (1996), pp. 336-338.

${ }^{26}$ BIBAS (2004), pp. 2542-2543.

${ }^{27}$ REDLICH et al. (2017a), p. 347.

${ }^{28}$ REDLICH et al. (2017a), p. 347.

${ }^{29}$ NIEVA (2019), p. 330.

${ }^{30}$ REDLICH et al. (2017a), p. 348

${ }^{31}$ GARCÍA (1995), pp. 92-93. 
estrategia de defensa, a guardar silencio, a la confrontación con la acusación y a la imposibilidad de recurrir la condena ${ }^{32}$.

Un aspecto que se ha puesto de manifiesto por la literatura es si el condenado es consciente que la sentencia de conformidad conlleva además de las consecuencias directas (la pena de multa, prisión u otras), las colaterales (o collateral attacks) ${ }^{33}$ que son las otras consecuencias aparejadas a la pena principal, como pueden ser las restricciones de empleo y licencias, la elegibilidad para vivienda pública o becas para estudiantes ${ }^{34}$. La investigación criminológica ha puesto de manifiesto, por ejemplo, que el hecho de poseer antecedentes penales puede conllevar consecuencias muy negativas para el futuro de la persona tanto en su ámbito personal, familiar y laboral, constituyendo las denominadas "penas invisibles" que sumadas a las penas principales dificultan, en última instancia, la reinserción ${ }^{35}$.

Aquí, igualmente radica la importancia de la dirección letrada, al ser los abogados los que acompañan y asesoran a los acusados en la toma de esta decisión ${ }^{36}$; ya que además de explicar las posibles consecuencias aparejadas al acuerdo de conformidad, deberían asegurar que sus clientes entienden los derechos a los que están renunciando y los efectos que a corto y largo plazo tiene su renuncia. Pero no solo los abogados, algunos autores defienden que los jueces deben verificar el conocimiento por parte de los acusados tanto de las consecuencias directas de la decisión que adoptan (los cargos y sanción penal), como de las consecuencias colaterales que implica esta decisión adoptada ${ }^{37}$.

De este modo, los acusados deberían tener información clara acerca de las consecuencias a las que se enfrentarán; necesitan saber no solo los términos de la pena impuesta sino también si pueden perder la custodia de sus hijos, si se les prohibirá trabajar en su profesión o si serán deportados $^{38}$. En general, los estudios demuestran que los acusados normalmente no son conscientes de las consecuencias colaterales aparejadas a la declaración de culpabilidad ${ }^{39}$.

Por otra parte, la decisión por la que el acusado acepta el acuerdo de conformidad debe ser voluntaria, tomada por el acusado de manera independiente y sin mediar amenaza o coerción. La duda recae sobre cuándo se puede afirmar que ha mediado algún tipo presión para aceptar una conformidad, ya que si el acuerdo alcanzado con el fiscal es muy ventajoso para el acusado o la potencial pena impuesta tras un juicio es demasiado gravosa se puede cuestionar si el acusado ha tenido otra opción para elegir que no sea conformarse ${ }^{40}$. En este sentido, como se expondrá más adelante, se abre la puerta a la posible condena de individuos inocentes, que ante el delicado y estresante contexto en el que se encuentran pueden decidir conformarse para evitar una condena mayor.

La decisión por la que se acuerda una conformidad también debe ser inteligente, el acusado debe ser capaz de entender y comprender los términos de la conformidad, las implicaciones que conlleva su decisión a corto y largo plazo ${ }^{41}$. No obstante, la escasa competencia legal de los acusados unido al empleo generalizado de terminología jurídica durante los procesos legales, incluido el acto de la conformidad, dificulta que esta toma de decisión sea razonada.

Asimismo, mientras que teóricamente el trámite de un coloquio oral es más o menos estándar y obligatorio en todas las jurisdicciones, en Estados Unidos el uso de los formularios

\footnotetext{
${ }^{32}$ REDLICH Y BONVENTRE (2015), p. 162; REDLICH et al. (2017b), p. 2.

33 Un ejemplo de ello es el listado realizado por la American Bar Association (Colegio de Abogados de los Estados Unidos) de las posibles consecuencias colaterales a las que los condenados podían enfrentarse, habiendo recogido más de 40.000 . Esta investigación que recibe el nombre de National Inventory of Collateral Consequences of Conviction es parte del National Reentry Resource Center, un proyecto fundado por el Departamento de Justicia de Estados Unidos, Bureau of Justice Assistance. https://www.americanbar.org/groups/criminal_justice/niccc/.

${ }^{34}$ REDLICH et al. (2017b), p. 2.

${ }^{35}$ LARRAURI (2015), p. 154.

${ }^{36}$ STEPHEN et al. (2012), p. 212.

${ }^{37}$ ROBERTS (2009), pp. 119-194.

38 BIBAS (2011b), p. 82.

${ }^{39}$ REDLICH Y BONVENTRE (2015), p. 171.

${ }^{40}$ DRIPPS (2015), p. 1364; SCHNEIDER (2018), pp. 20-21.

${ }^{41}$ RODRÍGUEZ-ARIAS (2019), p. 99
} 
escritos (tender of plea form) varía según la jurisdicción de cada estado, por ello, existen algunas investigaciones que se han centrado en examinar el contenido de estos documentos legales. Los tender of plea form a menudo contienen términos y un lenguaje con una gran complejidad técnica que exceden de la capacidad de las capacidades de la mayoría de los acusados En este sentido, Redlich y Bonventre realizaron una evaluación de la comprensión lectora necesaria para entender los plea forms disponibles en juzgados de este país, y hallaron que, en promedio, los formularios requerían de un nivel de lectura correspondiente a 110 y $12^{\circ}$ grado ${ }^{42}$, sin embargo, la mayoría de los delincuentes tienen una comprensión lectora correspondiente a 60 curso. Así, menos del $5 \%$ de los formularios examinados se podían entender con una comprensión lectora correspondiente a este nivel.

Estos resultados ponen de manifiesto que algunos acusados, a pesar de ser imputables, carecen de la competencia legal necesaria para seguir correctamente el coloquio judicial y los documentos escritos. En este sentido, Harlow revela que alrededor de dos tercios de los acusados no han terminado la escuela secundaria y muchos de ellos tienen problemas cognitivos y de salud mental ${ }^{43}$. Como podría esperarse, la capacidad de comprensión oral y auditiva de los acusados es bastante pobre, lo que conlleva importantes consecuencias negativas para el acusado ya que el método principal para determinar si la decisión del acusado cumple con los requisitos legales es a través del coloquio judicial.

Además, puesto que los acusados adoptan decisiones con limitada información y escaso entendimiento de dicha información ${ }^{44}$, la evidencia sugiere que los tribunales no inspiran demasiada confianza en su habilidad para reconocer y tratar estas deficiencias de los acusados en la comprensión del proceso de conformidad.

\section{Naturaleza del procedimiento de la conformidad: ¿descuento o castigo?}

La principal ventaja a la que aspira el imputado cuando se conforma es la de conseguir una reducción de la pena en la sentencia. Esta diferencia en la pena de la sentencia de conformidad con respecto a la sentencia obtenida tras un juicio se le suele denominar descuento (o reducción) y recompensa al acusado por el remordimiento demostrado al reconocer su culpabilidad. No obstante, no es un punto de vista compartido de manera unánime, ya que otro sector de la doctrina opina que este descuento equivale más bien a un castigo para aquellos que ejercen su derecho a un juicio justo ${ }^{45}$.

Los términos "descuento" y "penalización o castigo" connotan una opinión muy diferente; por esta razón, algunos autores ${ }^{46}$ hacen referencia a las diferencias en los hechos, los cargos y la pena definitiva tras la conformidad del acusado como "diferencia en la sentencia" (sentence differential).

Otros autores como Leverick (2004) argumentan que, independientemente de si se opina que esta diferencia es un descuento o una penalización, el acusado sufre el mismo destino, obteniendo una sentencia de condena. Además, surge la pregunta de si el acusado está obteniendo un descuento en la pena fruto de haber llegado a un acuerdo o si previamente a la negociación se había "inflado" la pena en el escrito de acusación del fiscal para dar la apariencia de "descuento" resultado de esa negociación de la conformidad. Como afirma Bibas (2004), los descuentos y las sanciones, así como las nociones de "pérdida y ganancia" son conceptos maleables, esto significa que pueden existir diferentes puntos de vista y, en consecuencia, es importante conocer cómo entiende el propio acusado esa diferencia en la sentencia.

En cualquier caso, existen dos principales modelos teóricos que tratan de explicar la verdadera naturaleza del instituto de la conformidad, manteniendo dos posturas claramente diferenciadas; así, mientras que desde el modelo de la sombra del juicio se entiende la diferencia

${ }^{42}$ REDLICH Y BONVENTRE (2015), pp. 166-167.

${ }^{43}$ HARLOW (2003), p. 9.

${ }^{44}$ BIBAS (2011), p. 80.

${ }^{45}$ DARBYSHIRE (2000), p. 901.

${ }^{46}$ SMITH (1987), p. 951; TATA Y GORMELY (2016), pp. 1-3. 
de pena en la sentencia de conformidad como un "descuento", desde el modelo de castigo del juicio se sostiene que consiste en una "penalización".

\subsection{La diferencia en la pena entendida como "descuento"}

Como se ha dicho anteriormente, los autores que defienden que la principal ventaja obtenida con la conformidad es una rebaja de la pena se amparan en la teoría de la conformidad bajo la sombra del juicio (en inglés, bargaining in the shadow of trial) para defender esta postura. Esta teoría que fue elaborada por Mnookin \& Kornhauser en 1979 se fundamenta en el principio de que los acusados adoptan decisiones racionales, y de acuerdo con este modelo, el acusado y el abogado considerarán aceptar el trato de conformidad o proceder a ir a juicio según las expectativas que tengan de lo que pueden obtener siguiendo cada camino ${ }^{47}$.

Los acusados racionales deberían aceptar entonces aquel acuerdo de conformidad cuando este contenga un mejor acuerdo (una medida menos lesiva) que el esperado en un juicio ${ }^{48}$.

Los fiscales haciendo uso de su poder de discrecionalidad, podrán ofrecer el "descuento" que quieran en cada caso, siendo conscientes de que un acusado racional solo aceptará el acuerdo si es más ventajoso que la sentencia que pueda obtener en juicio. Siendo esto así, Alschuler opina que este patrón es el resultado de un esfuerzo coercitivo ejercido por los fiscales para forzar a los acusados a declararse culpables y conformarse ${ }^{49}$. En su opinión, los fiscales que intentan evitar la celebración del juicio reducirán la pena del acuerdo por debajo del punto de equilibrio para forzar a los acusados a conformarse.

Igualmente, los defensores de la existencia de "descuentos" fruto de las conformidades sostienen que estas rebajas en los acuerdos con el fiscal serán mayores cuando la evidencia en contra del acusado sea más débil, como así se ha demostrado en investigaciones realizadas tanto en casos escenario ${ }^{50}$, como en la evaluación de casos reales ${ }^{51}$.

\subsection{La diferencia en la pena entendida como "castigo"}

En el bando opuesto se encuentran los autores que defienden que la naturaleza del instituto de la conformidad es premiar al acusado que decide conformarse y penalizar con una condena mayor a aquél que decida rechazar llegar a un acuerdo e ir a juicio. Los autores que defienden esta idea se amparan en la teoría del castigo del juicio elaborada por Eisenstein y Jacob en 1977, concebida como una alternativa a la teoría de sombra del juicio, y construida sobre principios diferentes.

Este modelo parte de la creencia de que jueces, fiscales y abogados forman parte de un grupo de trabajo interdependiente que trabaja hacia un objetivo compartido que es el de mantener la eficiencia del sistema judicial y aminorar costes procesales. De esa manera, una extensa literatura científica sugiere que los juzgados pueden valorar imponer "castigos" a aquellos acusados que ejercen su derecho a ir a juicio y no aceptan el acuerdo propuesto por el grupo de trabajo ${ }^{52}$. Por el contrario, si los acusados han aceptado un acuerdo de conformidad entonces recibirán medidas más leves.

Según este planteamiento, el acusado que insiste en no conformarse con la acusación es considerado como alguien que no actúa de manera razonable y que se arriesga a un juicio cuya celebración podría haber evitado. Existen dos variables relacionadas con el tamaño de ese "castigo" que tendrá la sentencia obtenida en juicio. La primera variable se relaciona con la

\footnotetext{
47 BIBAS (2004), pp. 2464-2467.

${ }^{48}$ BUSHWAY et al. (2014), p. 724.

${ }^{49}$ ALSCHULER (1968), pp. 64-65

${ }^{50}$ BUSHWAY et al. (2014), p. 735; KRAMER et al. (2007), p. 576

${ }^{51}$ KRAMER Y ULMER (2002), p. 906.

52 JOHNSON (2005), p. 780; ULMER Y BRADLEY (2006), pp. 650-652.
} 
evidencia del caso; parece haber cierto consenso de que la pena aumenta cuando existen pocas dudas acerca de la culpabilidad del acusado y aun así éste no se conforma; sin embargo, si existen dudas significativas y la evidencia es más débil, los miembros del grupo de trabajo pueden empatizar más con aquel que ejerce su derecho a juicio. La segunda variable hace referencia a la gravedad del delito; si el delito cometido es grave y va acompañado de una pena mayor, se entiende que hay más en juego y los profesionales entenderán al acusado que decida ir a juicio.

Más tarde, Steffensmeier, Kramer y Streifel (1993) y Steffensmeier, Ulmer y Kramer (1998) propusieron por primera vez la teoría de la "focal concerns" que integra la perspectiva del grupo interdependiente de trabajo proveniente de la teoría del castigo del juicio y trata de explicar también la naturaleza de la conformidad.

Se parte de la premisa de que los jueces generalmente cuentan con poca información de los infractores ${ }^{53}$, por lo que con el fin de reducir la incertidumbre de los casos penales acuden a recursos cognitivos que hacen más eficiente este proceso de toma de decisiones ${ }^{54}$. Este modelo sostiene que los fiscales y los jueces, a la hora de tomar sus decisiones, valoran tres aspectos esenciales: la culpabilidad de la persona acusada, la protección de la comunidad y las consecuencias prácticas (por ejemplo, limitaciones de recursos).

En primer lugar, la culpabilidad abarca varios factores entre los que se encuentran la gravedad del delito cometido, el daño a la víctima y los antecedentes penales. La literatura científica demuestra que los jueces y fiscales pueden negociar los cargos durante el proceso de la conformidad para garantizar que la pena realmente se ajuste al delito cometido ${ }^{55}$.

Al mismo tiempo, los fiscales y jueces pueden considerar que el estatus de "delincuentes primarios" es importante para la consideración del grado de culpabilidad. Así es posible que los actores judiciales perciban algunas acciones de algunos acusados como tan solo el resultado de una mala decisión. En estos casos, el uso de la conformidad sería visto como una herramienta para evaluar y distribuir la autoría y culpar de manera responsable en función del nivel de participación en el delito y el tipo de delincuente ${ }^{56}$.

En segundo lugar, según la teoría de la "focal concerns", los actores judiciales tienen en cuenta la protección de la comunidad, valorando algunos factores como la peligrosidad y la reincidencia ${ }^{57}$. Asimismo, los jueces basan sus decisiones en variables legales (gravedad del delito, reincidencia, etc.) como extralegales (género, situación familiar, etc.). En el caso concreto de Estados Unidos, la raza del acusado es una variable que recibe mucha atención por parte de la literatura científica. Según esta teoría, dada la percepción de que los acusados de raza negra e hispanos representan un mayor peligro para la comunidad, estos reciben sentencias más duras que sus homólogos blancos ${ }^{58}$. Igualmente, los acusados no blancos pueden verse en desventaja ya que también es probable que se les ofrezca menos acuerdos de conformidad ${ }^{59}$.

En definitiva, no existe un consenso a la hora de definir la naturaleza del instituto de la conformidad, mientras que algunos autores defienden que gracias a esta los individuos obtendrán una rebaja en la pena, otros autores consideran que utilizan las posibilidades que ofrece la conformidad de graduar las penas solicitadas por la acusación para imponer el castigo que se considere oportuno en cada caso.

\section{5. ¿Qué influye en la toma de decisiones?}

Como cualquier otra toma de decisiones, optar por conformarse o no con el acuerdo del fiscal puede estar influido por una variedad de factores individuales, sesgos cognitivos y sociales

\footnotetext{
${ }^{53}$ CHENG (2016), p. 34; STANCU Y VARONA (2017), pp. 9-11.

${ }^{54}$ ALBONETTI (1991), pp. 255-259.

55 STEFFENSMEIER Y DEMUTH (2000), pp. 708-711.

${ }^{56}$ BURROW Y LOWERY (2015), pp. 219-220.

${ }^{57}$ STEFFENSMEIER Y DEMUTH (2000), pp. 708-711.

58 HARTLEY et al. (2007), p. 61.

${ }^{59}$ BURROW Y LOWERY (2015), pp. 219-220; SAVITSKY (2012), p. 135.
} 
que, a menudo, pueden exacerbar o mitigar estas expectativas, perjudicando la racionalidad de los acusados y, por lo tanto, tienen su presencia o ausencia tienen una gran capacidad para predecir los resultados con precisión. Las investigaciones realizadas hasta el momento identifican tres factores individuales que pueden afectar al acusado a la hora de decidir si conformarse o no: la sobreconfianza, la negación y la tendencia a infravalorar los costes futuros ${ }^{60}$. Asimismo, los acusados pueden ser vulnerables ante los mecanismos de persuasión y las técnicas de influencia social utilizados durante este proceso. Lo vemos con más detalle a continuación.

\subsection{Factores individuales}

La investigación científica ha puesto de manifiesto que a menudo los procesados (especialmente hombres jóvenes y con un bajo nivel educativo) tienen un exceso de confianza, son demasiado optimistas cuando predicen sus posibilidades de conseguir una sentencia favorable y conseguir ser absueltos ${ }^{61}$. Así, en los estudios basados en escenarios hipotéticos donde se manipula la variable de probabilidad de condena en juicio, se ha demostrado que, aun cuando se estima que la probabilidad de condena es casi segura (por ejemplo, un $90 \%$ ), un gran porcentaje de los participantes siguen dispuestos a ir a juicio, arriesgándose a una pena más severa si eran condenados culpables, tal como defiende el modelo de castigo del juicio ${ }^{62}$.

De igual modo, se ha demostrado que la decisión de conformarse puede estar influida por la negación sobre lo ocurrido. La primera respuesta de los acusados es negar los hechos, su culpabilidad, minimizar la ilicitud de los hechos o el daño causado ${ }^{63}$. Estos acusados tienden a sobreestimar sus posibilidades de absolución ya que piensan que los jueces compartirán esta perspectiva distorsionada ${ }^{64}$. Además, incluso los acusados inocentes tienden a mantener perspectivas distorsionadas e ingenuamente pueden creer que su inocencia será fácilmente evidente para los operadores jurídicos.

Por último, la tendencia de los individuos de infravalorar los costes futuros puede influir igualmente en esta decisión. Las personas, especialmente los delincuentes, valoran más sus circunstancias presentes que futuras, pensando más a corto plazo ${ }^{65}$. Como la evidencia científica ha demostrado, la impulsividad es una característica común entre los delincuentes ${ }^{66}$, lo que hace más difícil valorar con precisión los costes futuros de las decisiones. Esto puede comportar el acuerdo de conformidades pensando en la rebaja de la medida (entendida como ganancia) a corto plazo y descontar las consecuencias a largo plazo de la decisión como pueden ser el registro de antecedentes penales o la renuncia de derechos ${ }^{67}$.

Igualmente, los delincuentes con frecuencia perciben erróneamente los riesgos y están especialmente dispuestos a asumirlos, por esta razón, muchos de los acusados preferirán celebrar un juicio. De esta forma, según esta teoría, los acusados que mantienen una posición neutral, ante el riesgo, aceptarán acuerdos con una pena igual o menor que la esperada en juicio; un acusado con preferencias por el riesgo necesitará un descuento mayor en la pena para aceptar el acuerdo, mientras que un acusado adverso al riesgo podría aceptar el acuerdo aun cuando este contenga una pena que excede la esperada en juicio ${ }^{68}$.

Normalmente, los acusados perciben su libertad como su estado natural por lo que cualquier sentencia de condena será percibida como una pérdida. No obstante, para aquellos que están detenidos de manera preventiva esperando la celebración del juicio pueden ser más propensos a ver el encarcelamiento como "el estado natural" y la libertad eventual como una

${ }^{60}$ REDLICH et al. (2017b), p. 5.

61 BIBAS (2004), p. 2500.

62 BORDENS (1984), pp. 60-63.

63 BUSHWAY et al. (2014), pp. 732-751.

${ }^{64}$ BIBAS (2004), pp. 2498-2502.

${ }^{65}$ FOUNTAIN (2017), p. 72.

66 GOTTFREDSON Y HIRSCHI (1990), p. 90.

${ }^{67}$ REDLICH et al. (2017a), p. 346.

${ }^{68}$ REDLICH et al. (2017b), p. 5. 
ganancia ${ }^{69}$. Así se expone en el estudio de Kellough y Wortley (2002) que, basándose en una revisión de expedientes judiciales reveló que los acusados que se encontraban en prisión preventiva tenían 2,5 veces más probabilidades de declararse culpables que aquellos que se encontraban en libertad ${ }^{70}$.

\subsection{Tácticas de influencia social}

La decisión de declararse culpable y conformarse refleja, pues, un conjunto de sesgos cognitivos que poseen los imputados, pero también pueden intervenir sobre ellos tácticas de influencia social que interactúan con estos factores individuales. Las tácticas de influencia social pueden ser muy útiles en las negociaciones de la conformidad para alentar a los acusados a declararse culpables.

En primer lugar, de acuerdo con el principio de reciprocidad ${ }^{71}$, las personas están más dispuestas a cumplir cuando el ofertante ha proporcionado antes una concesión. En el ámbito de las negociaciones de la conformidad, a cambio de concesiones procesales (respecto a la duración de las penas, el número de cargos...), los acusados aceptan declararse culpables y renunciar a sus derechos. Además, los acusados se pueden ver influenciados por el tamaño de esa concesión (una gran rebaja de la pena, por ejemplo) en el trato con el fiscal, de modo que cuando esos tratos se vean más ventajosos, las personas estarán más dispuestas a declararse culpables.

En segundo lugar, los acusados también pueden verse influidos por el principio de la validación social, según el cual, las personas evalúan sus situaciones en el contexto de lo que otros han hecho en situaciones similares o, en este caso, de lo que se les ha ofrecido. Los acusados, inseguros de qué decisión tomar, confían en la evaluación y opinión que tiene el abogado o la familia del acusado sobre el acuerdo de conformidad ${ }^{72}$.

Otra de las tácticas de influencia social reside en el principio de la escasez ${ }^{73}$, basado en el miedo que genera la situación de saber que si uno no actúa rápidamente, la oportunidad de obtener algo que se desea desaparecerá rápidamente. En este caso, los acusados son conscientes de que la posibilidad de llegar a un acuerdo de conformidad tiene un tiempo limitado y los fiscales pueden presionar a los acusados para que acepten este acuerdo.

Finalmente, también puede incidir el principio de autoridad, según el cual las personas tienden a cumplir con las peticiones o lo ordenado por personas en puestos de autoridad. En virtud del estatus profesional, los fiscales, jueces y abogados tienen una autoridad considerable y sus recomendaciones tienen un peso significativo. Desde el punto de vista del acusado, la recomendación que es hecha por una persona que tiene mayores conocimientos legales y comprende mejor el sistema judicial, puede predecir mejor el resultado de un juicio y las consecuencias futuras, por lo tanto, el acusado confiará en su recomendación. La cultura propia de cada tribunal se cree que impulsa muchas de estas diferencias en la forma de proceder con las conformidades, y este proceder puede variar significativamente entre los distintos sistemas judiciales; por ello es importante conocer y documentar empíricamente cómo se ve afectado el proceso de toma de decisiones en cada juzgado según la rutina de cada juzgado ${ }^{74}$.

\section{El dilema de la conformidad del inocente}

Desde que comenzó a instaurarse en el siglo XIX el mecanismo de la conformidad, la comunidad científica se ha preocupado por el riesgo de que acusados inocentes acepten acuerdos de conformidad. Si partimos de la base de que contamos con un sistema de justicia

\footnotetext{
69 BIBAS (2004), p. 2544.

${ }^{70}$ KELLOUGH Y WORTLEY (2002), p. 199.

${ }^{71}$ CIALDINI (2009), pp. 20-24.

72 VILJOEN et al. (2005), p. 255.

${ }^{73}$ CIALDINI (2009), pp. 204-205.

${ }^{74}$ REDLICH et al. (2017b), p. 8.
} 
penal garantista, debemos pensar que es muy improbable que un inocente se proclame como culpable al inicio de un proceso penal.

Sin embargo, según revelan los autoinformes, las tasas de declaraciones de culpabilidad falsas varían desde el $18 \%$ de los delincuentes juveniles ${ }^{75}$, al $37 \%$ entre los delincuentes con enfermedades mentales ${ }^{76}$. En resumen, hay razones para creer que el número de declaraciones de culpabilidad falsas es mucho mayor del que se conoce actualmente ${ }^{77}$, aunque no es posible hacer una estimación real de la tasa de personas inocentes que se conforman.

Cuando se alcanza una conformidad, se desvirtúa el principio de la presunción de inocencia, asumiendo el acusado su culpa y recibiendo una sentencia de condena; por lo tanto, si con la conformidad se puede conducir a la condena de personas inocentes, se lesionarían los derechos a la presunción de inocencia, al honor del acusado o al mandato de resocialización de las penas privativas de libertad (si hubiese), es decir, se atentaría contra principios constitucionales básicos y se generaría un inadmisible reproche desde el punto de vista constitucional $^{78}$.

La literatura científica ha demostrado que la dinámica estructural del sistema penal perjudica a los acusados que son inocentes, quienes, en caso de riesgo de una pena mayor, que podría ser impuesta en el juicio, prefieren la pena menor acordada en la negociación con el fiscal ${ }^{79}$, siendo preferible una pena leve y personalmente asumible que enfrentarse al riesgo de ser condenado con una pena más grave y difícil de asumir.

Por esta razón, siendo conscientes de esta problemática, en las últimas décadas Neufeld \& Scheck fundaron una organización dedicada a la utilización de las pruebas ADN como medio para demostrar la inocencia de individuos que fueron condenados injustamente ${ }^{80}$. Esta iniciativa surgida en Estados Unidos y replicada en distintos países, ha brindado la oportunidad de investigar también cómo personas inocentes pueden ser condenadas a través de la conformidad. A pesar de las divergencias que existen entre el modelo de proceso penal estadounidense y el resto de los sistemas procesales, podría pensarse que una situación parecida podría replicarse en cualquier sistema judicial ya que este peligro no deriva solo del modelo penal sino también de la posibilidad de someter a negociación la aceptación de culpabilidad del acusado sin un control eficaz por parte del tribunal, una situación generada en la mayoría de los procesos penales ${ }^{81}$.

En este sentido, los estudios basados en casos escenario sugieren que las personas inocentes están sujetas a otros sesgos que pueden llevarlos a abordar la toma de decisiones de manera diferente a las personas culpables, una tendencia que se ha acuñado como el "efecto de inocencia" ${ }^{82}$. Estos acusados inocentes creyendo que su inocencia será evidente para los actores judiciales, les conducirá a evaluaciones demasiado optimistas de sus probabilidades de absolución y, en consecuencia, pueden ignorar las tasas de condena en juicio, creyendo que esa tasa alta de condena no se aplica a ellos.

Así, estudios previos realizados con muestras de estudiantes ${ }^{83}$ indican que los participantes "inocentes" prefieren arriesgarse e ir a juicio ya que aceptar haber cometido un delito que no han cometido es percibido como injusto (resistencia ante la injusticia). Igualmente, los individuos culpables deberían ser más propensos a percibir la condena y el castigo como el resultado natural de su delito mientras que las personas inocentes deberían percibir cualquier castigo como una pérdida inmerecida ${ }^{84}$.

\footnotetext{
${ }^{75}$ MALLOY et al. (2014), p. 186.

${ }^{76}$ REDLICH et al. (2010), p. 87.

${ }^{77}$ REDLICH (2010), p. 957.

78 TATA Y GORMLEY (2016), pp. 21-22; LASCURAÍN Y GASCÓN (2018), pp. 7 y 20.

79 LASCURAÍN Y GASCÓN (2018), p. 13; FERRÉ (2018), p. 10.

${ }^{80}$ Este proyecto es conocido como Innocence Project creado en el año 1992.

${ }^{81}$ LASCURAÍN Y GASCÓN (2018), p. 7.

82 GAZAL-AYAL Y TOR (2012), p. 339

83 GREGORY et al. (1978), p. 1525; BORDENS (1984), p. 66; TOR et al. (2010), pp. 113-114; SCHNEIDER (2018), pp. 19-20.

${ }^{84}$ KAHNEMAN Y TVERSKY (1979), p. 286.
} 
Los principales modelos teóricos (bajo la sombra del juicio y castigo por el juicio) no son capaces de predecir el comportamiento de un acusado inocente, así desde la perspectiva del modelo bajo la sombra del juicio, lo único que importa es la probabilidad de condena, por lo que las personas inocentes, si son verdaderamente racionales, deberían declararse culpables siempre que la oferta del fiscal será inferior a la pena esperada en juicio ${ }^{85}$. Esta es la razón por la que algunos autores ${ }^{86}$ consideran que estas ofertas son coercitivas, dado que personas racionales e inocentes se podrán declarar culpables en algunos casos cuando el descuento sea suficientemente grande ${ }^{87}$.

Igualmente, se ha demostrado que la tasa de individuos inocentes aumenta sustancialmente en ciertas circunstancias, si el acuerdo de la conformidad contiene una pena mucho menor que la esperada en una condena en juicio ${ }^{88}$ si la posible pena en juicio es una privación de libertad, si el acusado se encuentra en prisión preventiva antes de ir a juicio ${ }^{89}$, si se enfrenta a la pena de muerte en caso de perder un juicio o si el acusado hubiera asumido su culpa previamente en comisaría ${ }^{90}$.

Además, existen otras razones para dudar de la suposición de que es improbable que un acusado inocente se declare culpable. Así, desde el punto de vista del acusado inocente la conformidad puede suponer un modo de evitar los costes de índole moral; se elimina el estrés y sufrimiento propio que acarrea el proceso penal y se evitan los costes derivados del juicio y las repercusiones negativas sobre la vida personal y familiar ${ }^{91}$.

De manera similar, el acusado buscará en la conformidad la forma de evitar costes a terceros; se trata más bien de la intención de proteger a alguien querido de unas consecuencias más severas. Esta idea coincide con hallazgos previos que demuestran que la mayoría de las confesiones falsas reportadas fueron hechas con el fin de proteger a un tercero ${ }^{92}$.

Por último, la literatura científica ha puesto de manifiesto las circunstancias y características propias de algunos colectivos (los menores de edad, las personas con discapacidad intelectual y las que poseen alguna enfermedad mental) que los convierte en vulnerables para ceder ante la presión de la conformidad ${ }^{93}$.

En relación con la minoría de edad, la inmadurez e impulsividad propia de los jóvenes ${ }^{94}$, la mayor susceptibilidad de sufrir estrés y tensión ${ }^{95}$, la falta de experiencia en el trato con las instituciones penales y escasa competencia legal ${ }^{96}$, la tendencia a cumplir y obedecer las órdenes dadas por un autoridad ${ }^{97}$, la preferencia del riesgo, la influencia de los iguales ${ }^{98}$ y la escasa orientación futura ${ }^{99}$ justifican que sea necesario desarrollar investigaciones centradas exclusivamente en la toma de decisiones de los menores de edad.

En definitiva, a pesar de que parezca inconcebible que un individuo inocente consienta obtener una sentencia de condena a través de su conformidad con el escrito de la acusación, existen investigaciones empíricas que así lo evidencian, demostrando que la acusación cuenta con distintas artimañas que influyen en el proceso de toma de decisión del acusado, cuestionando así la calidad y la legitimidad de la justicia.

\footnotetext{
${ }^{85}$ REDLICH et al. (2017b), p. 6.

${ }^{86}$ SCHULHOFER (1992), pp. 70-74; VIANO (2012), p. 114

${ }^{87}$ GAZAL-AYAL Y TOR (2012), p. 379.

${ }^{88}$ BORDENS (1984), pp. 65-71.

${ }^{89}$ BORDENS (1984), p. 60.

90 GAZAL-AYAL Y TOR (2012), p. 387.

${ }^{91}$ LASCURAÍN Y GASCÓN (2018), p. 16.

92 VILJOEN et al. (2005), p. 265; MALLOY et al. (2014), p. 189.

${ }^{93}$ FLYNN Y FREIBERG (2018), p. 187; HELM et al. (2018), pp. 22-28, entre otros.

${ }^{94}$ LAMONTAGNE (2013), p. 56.

${ }^{95}$ LAMONTAGNE (2013), p. 56

${ }^{96}$ GRISSO et al. (2003), pp. 358-361.

${ }^{97}$ LAPP (2017), p. 916.

${ }^{98}$ GARDNER Y STEINBERG (2005), pp. 626 y 634.

${ }^{99}$ CAUFFMAN et al. (2010), pp. 204-206.
} 


\section{Percepciones de justicia en el proceso de la conformidad}

Existe una amplia literatura científica que destaca la importancia de la confianza de la comunidad en el sistema de justicia penal y de la percepción por parte de la ciudadanía de que el sistema y las actuaciones sean legítimas. Sin dicha confianza, respeto y legitimidad, el sistema de justicia no funciona con éxito y muchos ciudadanos no cooperarán con el sistema de justicia. Esta teoría conocida como justicia procedimental destaca que el sistema puede garantizar el cumplimiento de la ley solo si se considera justo, imparcial y legítimo.

El autor de esta teoría Rob Tyler identificó cuatro factores que afectan a la forma en la que la comunidad evalúa el sistema judicial ${ }^{100}$ : en primer lugar, las personas quieren tener una "voz" en el proceso de toma de decisiones y poder exponer su versión del caso ante las autoridades, es decir, se debe constatar si las personas involucradas tienen la oportunidad de constar su versión de la historia; en segundo lugar, las personas obedecen a las autoridades si se percibe su neutralidad (sus decisiones están basadas en principios legales en lugar de opiniones personales y prejuicios); en tercer lugar, las personas demandan ser tratadas con dignidad y respeto; y finalmente, los individuos valoran que las autoridades se muestren como individuos en los que se puede confiar. En definitiva, la percepción de la voz, la neutralidad, la confiabilidad y el respeto pueden promover la aceptación de decisiones que de otro modo se considerarían incorrectas o sustancialmente injustas.

La investigación social indica que la implementación de las normas de justicia procedimental no solo puede aumentar la satisfacción del acusado con el resultado de la negociación con el fiscal y de la sentencia de conformidad, sino que también puede contribuir a la legitimidad percibida del sistema de justicia penal y, en última instancia mejorar el cumplimiento voluntario de las normas legales ${ }^{101}$. En resumen, se cree que una mayor atención por parte de las autoridades legales en términos de justicia procedimental puede aumentar la cooperación de los acusados durante su paso por el proceso penal y disminuir su reincidencia.

De esta forma, en la medida que las actuaciones legales y sus interacciones con los ciudadanos son tal relevantes para el buen funcionamiento del sistema, surge la pregunta de hasta qué punto el proceso por el que se adopta la decisión de conformarse puede afectar a las percepciones de justicia procedimental de los acusados. Así, estos cuatro factores de la justicia procedimental se pueden emplear también para valorar la práctica de la conformidad, en tanto que van a contribuir en última instancia al aumento de la confianza en la justicia por parte de los individuos que estén inmersos en estos procesos penales, incrementando también la legitimidad institucional y el cumplimiento futuro de la ley ${ }^{102}$.

En primer lugar, el proceso por el que se llega a la conformidad puede permitir que los acusados tengan voz en la medida en que perciben una oportunidad para poder contar su historia. Curiosamente, algunas investigaciones sugieren que incluso si la oportunidad de ser escuchado se produce después de haber tomado la decisión relevante, los individuos valoran haber tenido esa voz $^{103}$; sin embargo, los efectos positivos son mayores si se brinda esta oportunidad de ser escuchado antes de finalizar el proceso. Por ello, para garantizar buenas percepciones de justicia procedimental en los procedimientos de conformidad los fiscales, deberían asegurar dar al acusado esta oportunidad para hablar antes de presentar una oferta al acusado. Igualmente, el abogado, en la medida en que se considera el garante de esa "voz" del acusado, no debe pactar un acuerdo de conformidad con el fiscal sin haber consultado con su

\footnotetext{
100 TYLER (2006), p. 76.

101 TYLER (1990), pp. 19-22.

102 TYLER (1990), pp.19-22 y JACKSON et al. (2012), pp. 1051-1071, entre otros, han abordado este tema con gran profundidad, señalando que la imparcialidad de la toma de decisiones (neutralidad e independencia de las autoridades) y la calidad del trato interpersonal (respeto por parte de las autoridades y facilitar la participación en el proceso) son dos aspectos que la sociedad valorará para considerar que un procedimiento es justo. De esta forma, si la sociedad percibe imparcialidad, un trato respetuoso y posibilidad de participación, aumentará la confianza en las autoridades, reconociendo a estas como legítimas. Por ello, la ciudadanía reconocerá su autoridad y obedecerá las normas dictadas por estas, considerándolas moralmente apropiadas.

${ }^{103}$ O'HEAR (2008), pp. 426-427.
} 
cliente tal propuesta ${ }^{104}$. En este sentido, se han hallado correlaciones significativas positivas entre la percepción del acusado en términos de justicia procedimental y la cantidad de tiempo que pasaba reuniéndose con su letrado ${ }^{105}$ y la calidad de trato y satisfacción con el letrado ${ }^{106}$.

En segundo lugar, la neutralidad está asociada con la imparcialidad y la honestidad. La mayoría de los fiscales se presumen neutrales, pero deben convencer y asegurar a los acusados que realmente lo son. En el ámbito de la conformidad, los fiscales podrían mejorar la perspectiva de los acusados explicando a los acusados los pasos seguidos para adoptar su posición, demostrando que se siguió un proceso neutral ${ }^{107}$. Además, aparte de que el abogado transmita esta información al acusado, el fiscal podría para mejorar las percepciones de justicia procedimental explicar su razonamiento en la audiencia del juicio, cuando el acusado esté físicamente presente para aceptar la conformidad.

En tercer lugar, las personas infieren confianza cuando creen que las autoridades se preocupan por su bienestar y quieren tratarlas de manera justa. Los acusados percibirán esa confianza si las autoridades demuestran que han tenido en cuenta la información ofrecida por el acusado. En el contexto de la conformidad, el fiscal debe abordar expresamente cualquier reclamo presentado por el acusado en aras de conseguir un trato más indulgente, pudiendo contestar en los propios términos del acusado por qué acepta o rechaza ese trato ${ }^{108}$.

En cuarto lugar, los individuos valoran positivamente que las autoridades les traten con respeto y educación, garantizando los aspectos emocionales ${ }^{109}$. Igualmente, $\mathrm{O}^{\prime}$ hear recomienda que los fiscales deben evitar, algo que es frecuente en la práctica, realizar ofertas de conformidad al acusado cuando no dejan un tiempo efectivo para que este pueda tener tiempo suficiente para valorarlo con su abogado.

En definitiva, las percepciones de justicia procedimental podrían aumentar durante la celebración de esta práctica con simples recomendaciones: asegurar que el acusado ha tenido la oportunidad de contar su versión de la historia, desarrollar criterios objetivos que guíen la negociación de la acusación y defensa, explicar los motivos que reconocen un trato más indulgente y abstenerse de las tácticas de presión.

\section{Conclusiones}

El instituto de la conformidad como forma de resolución negociada de conflictos penales comenzó siendo una solución excepcional para resolver de manera ágil los procesos penales surgidos en Estados Unidos en el siglo XIX y se ha expandido internacionalmente convirtiéndose en la actualidad en la principal vía de finalización del proceso penal en multitud de países. De esta forma, la administración de justicia puede obtener una sentencia de conformidad condenatoria- sin la necesidad de celebrar total o parcialmente la fase de enjuiciamiento; por lo tanto, se favorece la economía procesal y la practicidad, reduciendo la labor de la acusación, la defensa y el juez, resultando todos beneficiados.

De este modo, el acusador particular consigue mediante la sentencia de conformidad una condena con mayor brevedad para el acusado y obtendrá antes la reparación del daño causado mediante la pena de responsabilidad civil. De la misma manera, el Ministerio Fiscal se asegura la defensa y protección de los derechos de la ciudadanía sin la necesidad de probar de forma constatada la relación del hecho punible con el acusado, debido a que no hace falta conexión con las pruebas para establecer la culpabilidad o inocencia del acusado ${ }^{110}$. Por otro lado, se encuentra la ventaja a la que aspira el acusado a cambio de su conformidad es una rebaja de la pena, especialmente cuando con ello consigue una pena no privativa de libertad o una

\footnotetext{
104 O'HEAR (2008), p. 427.

${ }^{105}$ CASPER et al. (1988), p. 498.

106 PETERSON-BADALI (2007), p. 391

107 O'HEAR (2008), pp. 428-430.

108 O'HEAR (2008), pp. 428-430.

109 FERNÁNDEZ-MOLINA (2013), pp. 227-228

${ }^{110}$ FERRÉ (2018), p. 4.
} 
suspensión de la pena de prisión ${ }^{111}$. Puesto que parece que todos los actores legales y también el propio acusado obtienen ventajas de esta práctica, ningún legislador se ha planteado todavía limitar la conformidad mediante reformas legales.

El mecanismo de la conformidad ha contado con partidarios y detractores pertenecientes tanto al ámbito más conservador como al más liberal que abogan por la protección de los derechos humanos. Por un lado, el bando más conservador suele centrar sus críticas en la indulgencia de la sentencia obtenida tras la conformidad, ya que la pena impuesta al individuo es menor de la que "en justicia" se merece. Igualmente, priva a las víctimas y a la comunidad de su oportunidad de obtener justicia mientras se favorece al acusado.

Por otro lado, los pensadores liberales critican que se trata de una práctica perniciosa que lleva a los que carecen de recursos económicos a una condena conseguida de manera más fácil y eficiente, hasta el punto de que puedan ser condenados por delitos de los que son inocentes. Además, la presión ejercida por la acusación y la defensa para aceptar el acuerdo de negociación y evitar así el riesgo de una pena mayor, unido a las carencias propias de los imputados y las influencias sociales a las que pueden estar sometidos lleva a los individuos a una situación límite en la que terminan conformándose con una pena aparentemente menor, sin olvidar las consecuencias que en términos de justicia procedimental conllevará el cumplimiento de la condena.

Igualmente, a excepción de que la víctima se persone en el juicio como acusación particular, la negociación propia de la conformidad se lleva a cabo sin su participación, lo que conlleva que seguramente no se vean representados sus intereses. Además, existe asimetría en la información que las partes conocen; mientras que la fiscalía conoce el marco probatorio del que dispone en contra del acusado, la defensa no cuenta con los mismos medios.

Con todo ello se desprende que la conformidad resulta un "mal necesario" en nuestro sistema judicial actual, a pesar de que se entiende que produce una degradación de la justicia penal. De hecho, el mecanismo de la conformidad suscita quejas desde la perspectiva social, tanto porque la conformidad del acusado en relación a determinados delitos graves puede herir sensibilidades (los hechos no serán verdaderamente juzgados) como porque puede no tenerse en cuenta el eventual interés de la sociedad en la resolución de conflictos subyacentes. Además, permite la vulneración de numerosos principios procesales, ya que no se cumplen sus fines ni en relación con la sociedad (la pena puede ser insuficiente) ni al autor (se condena sin pasar por un juicio justo ${ }^{112}$. Además, esta negociación se caracteriza por su clandestinidad, generando un espacio de negociaciones paraprocesales entre fiscalía y defensa, normalmente carentes de transparencia.

El acusado, culpable o inocente, que cuenta con una baja competencia legal y está sometido a factores de estrés o nerviosismo se puede ver abocado a conformarse tanto por las ostensibles reducciones que la acusación ofrece como por el temor de una mayor pretensión jurídica por parte de la acusación si este desea ejercer su derecho a un juicio con todas las garantías.

Además, con la agilidad que proporciona el instituto de la conformidad se ha intentado evitar el colapso de los órganos judiciales, sin aportar los medios personales y materiales para ello y a costa de los derechos e intereses de los acusados. De este modo, resulta injustificable que se legitime el instituto de la conformidad amparándose en la ventaja de la rapidez (que no eficacia) en términos de economía procesal cuando se atenta contra los fundamentos más sólidos de un Estado de derecho.

Es necesario que nos replanteemos si estamos dispuestos a asumir las desventajas que plantea el uso de la conformidad en los diferentes modelos procesales donde se utiliza a diario, siendo quizás necesario reforzar el papel del juez para ejercer un control jurisdiccional del acuerdo llevado a cabo entre las partes, asegurando que la decisión del acusado es voluntaria, que no ha mediado ningún tipo de coacción, y que entiende todas las consecuencias que

${ }^{111}$ LASCURAÍN Y GASCÓN (2018), p. 5.

112 FERRÉ (2018), p. 9. 
conlleva. Igualmente, sería importante reforzar también algunos aspectos términos de justicia procedimental, ya que si el acusado percibe que ha recibido un trato digno, que puede confiar en los actores judiciales y en las instituciones y si ha podido participar durante el procedimiento, aceptará de mejor grado la sentencia de conformidad y se consolidará el cumplimiento futuro de la ley.

\section{BIBLIOGRAFÍA CITADA}

Albonetti, Celesta (1991): "An integration of theories to explain judicial discretion", en: Social Problems (Vol. 38), pp. 247-266.

AlsChUleR, ALbert W. (1968): "The prosecutor's role in plea bargaining", en: The University of Chicago Law Review (Vol. 36, № 1), pp. 50-112.

AlsChUleR, Albert W (1979): "Plea-bargaining and its history”, en: Columbia Law Review (Vol. 79, № 1), pp. 1-43.

BARONA VILAR, SILVIA (1994): "Algunas reflexiones en torno al instituto de la conformidad en el proceso penal", en: La Ley: Revista jurídica española de doctrina, jurisprudencia y bibliografía (№ 4), pp. 915-935.

BiBAs, StePHANOS (2004): "Plea Bargaining Outside the Shadow of Trial”, en: Harvard Law Review (Vol. 117, № 8), pp. 2463-2547.

BiBAS, StEPHANOS (2011a): "Regulating the plea-bargaining market: From caveat emptor to consumer protection", en: California Law Review (Vol. 99), pp.1117-1162.

BIBAS, StePHANOS (2011b): "The myth of the fully informed rational actor", en: Saint Louis University Public Law Review (Vol. 31), pp. 79-86.

BORDENS, KENNETH S. (1984): "The effects of likelihood of conviction, threatened punishment, and assumed role on mock plea bargaining decisions", en: Basic and Applied Social Psychology (Vol. 5, № 1), pp. 59-74.

BUSHWAY, SHAWN; REDLICH, AlLISON Y NORRIS, ROBERT J. (2014): "An explicit test of "bargaining in the shadow of trial."'” en: Criminology: An Interdisciplinary Journal (Vol. 52, № 4), pp. 723-754.

BURROW, JOHN D. Y LOWERY, PATRICK G. (2015): “A preliminary assessment of the impact of plea bargaining among a sample of waiver-eligible offenders" en: Youth violence and juvenile justice (Vol. 13, № 3), pp. 211-227.

CASPER, JONATHAN D.; TYLeR, TOM Y FISHER, BONNIE (1988): "Procedural justice in felony cases", en: Law \& Society Review (Vol. 22, № 3), pp. 483-508.

Cauffman, elizabeth; Shulman, elizabeth. P.; Steinberg, laurence; Claus, Eric; Banich, Marie T.; GRAHAM, SANDRA Y WoOlaRD, JeNNIFER (2010): "Age differences in affective decision making as indexed by performance on the lowa gambling task" en: Developmental Psychology (Vol. 46, № 1), pp. 193-207.

CHENG, KEVIN KWOK-YIN (2016): "Public approval of plea bargaining in Hong Kong: The effects of offender characteristics", en: International Criminal Justice Review (Vol. 26, № 1), pp. 31-48.

CIALDINI, ROBERT B. (2009): Influence: Science and practice (Boston, Allyn \& Bacon).

DARBYSHIRE, PENNY (2000): “The Mischief of Plea Bargaining and Sentencing Rewards", en: Criminal Law Review (Vol. 1), pp. 895-910.

Del Moral García, Antonio (2008): "La conformidad en el Proceso Penal (Reflexiones al hilo de su regulación en el ordenamiento español)", en: Revista Auctoritas Prudentium (Vol. 1), pp. 1-22. 
DEVERS, LINDSEY (2011): “Plea and charge bargaining: Research summary", en: BJA Report January. Disponible en: https://bja.ojp.gov/sites/g/files/xyckuh186/files/media/document/PleaBargainingResearchSum mary.pdf [visitado el 15 de agosto de 2019].

DRIPPS, DonALD A. (2015): “Guilt, Innocence, and Due Process of Plea Bargaining", en: William \& Mary Law Review (Vol. 57, № 4), pp. 1343-1393.

EASTERBROOK, Frank (1983): “Criminal procedure as a market system", en: The Journal of Legal Studies (Vol. 12, № 2), pp. 289-332.

EISENSTEIN, JAMES y JACOB, HERBERT (1977): Felony justice: An organizational analysis of criminal courts (Boston, Little, Brown).

FAIR TRIAL INTERNATIONAL (2016): "The disappearing trial. Towards a rights-based approach to trial waiver systems". Disponible en: https://www.fairtrials.org/sites/default/files/publication_pdf/Report-The-Disappearing-Trial.pdf [visitado el 01 de abril de 2020].

FeRnÁNDEZ MolinA, ESTHer (2013): “Una aproximación a la figura del abogado en la justicia de menores. Una aproximación a la figura del abogado en la justicia de menores", en: Cuadernos de Política Criminal (Vol. 1, № 109), pp. 217-242.

FerRÉ OlivÉ, JosÉ CARLOS (2018): “El Plea Bargaining, o cómo pervertir la justicia penal a través de un sistema de conformidades low cost", en: Revista Electrónica de Ciencia Penal y Criminología (№ 20), pp.1-30.

FlynN, Asher y Freiberg, Arie (2018): Plea negotiations: Pragmatic justice in an imperfect world (Bristol, Reino Unido, Springer).

FOUNTAIN, ERIKA N. (2017): Adolescent plea bargains: Developmental and contextual influences of plea bargain decision making (Washington, D.C, Tesis doctoral, Georgetown University).

Fraga MAndí́n, JaVIer. (2016): La sentencia de conformidad. Especial consideración de la denominada conformidad premiada (Salamanca, Tesis doctoral, Universidad de Salamanca).

Fraga Mandián, JaVIer. (2018): Las diversas manifestaciones de la conformidad en el Derecho procesal penal español (Madrid, Editorial Jurídica Sepín).

GARCía RodRíGUEZ, Nicolás (1995): “Aproximación al estudio de la justicia penal negociada de los EE. UU: The Plea Bargaining Process", en: REDEN: Revista española de estudios norteamericanos (№ 9), pp. 91-107.

GARDNER, MARGO Y SteinBerg, LAURENCE (2005): "Peer influence on risk taking, risk preference, and risky decision making in adolescence and adulthood: an experimental study", en: Developmental psychology (Vol. 41, № 4), pp. 625-635.

Gazal-Ayal, OREN y RIZA, LIMOR (2009): "Plea-bargaining and prosecution" en: Criminal Law and Economics (Cheltenham, UK, Nuno Garoupa)), pp. 1-41.

GAZAl-Ayal, Oren y ToR, Avishalom (2012): “The Innocence Effect”, en: Duke Law Journal (Vol. 62, № 2), pp. 339-401.

Gottfredson, Michael y HiRSCHI, Travis (1990): A general theory of crime (New York, Macmillan).

GRegory, W. LARRY; MOWEN, John C. y LINDER, DARWYN E. (1978): "Social psychology and plea bargaining: Applications, methodology, and theory", en: Journal of Personality and Social Psychology (Vol. 36, № 12), pp. 1521-1530.

GRIFFiths, CURT TAYLOR; KLEIN, JOHN F. y VeRdUn-JONES, SIMON NiCHOLAS (1980): Criminal justice in Canada: An introductory text (Canadá, Butterworth \& Company). 
Grisso, Thomas; Steinberg, lauren; Woolard, Jennifer; Cauffman, Elizabeth; Scott, Elizabeth; Graham, Sandra; Lexcen, Fran; Repuccl, N. Dickon y SChwartZ, Robert (2003): "Juveniles' competence to stand trial: A comparison of adolescents' and adults' capacities as trial defendants", en: Law and Human Behavior (Vol. 27, № 4), pp. 333-363.

HARLOW, CAROLINE WOLF (2003): Education and correctional populations. Bureau of Justice Statistics Special Report., Office of Justice Programs, 13 (Washington, DC, U. S. Department of Justice).

HARTLEY, RichaRd; MAdDAN, SEAN y SPOHN, CASSIA (2007): “Concerning conceptualization and operationalization: Sentencing data and the focal concerns perspective. A research note", en: Southwest Journal of Criminal Justice (Vol. 4, № 1), pp. 58-78.

Helm, Rebecca K.; Reyna, Valerie F.; Franz, Allison A. y Novick, Rachel Z. (2018): “Too young to plead? Risk, rationality, and plea bargaining's innocence problem in adolescents", en: Psychology, Public Policy, and Law (Vol. 24, № 2), pp.1-39.

INNOCENCE PROJECT (2008): "The Innocence Project". Disponible en: http://www.innocenceproject.org [visitado el 01 de abril de 2020].

Jackson, Jonathan; Bradford, Ben; Hough, Mike; Myhill, ANdY; Quinton, PAUL, y Tyler, TOM, R. (2012): "Why people comply with the Law? Legitimacy and the Influence of Legal Institutions", en: British Journal of Criminology (Vol. 52, № 6), pp.1051-1071.

JOHNSON, BRIAN (2005): “Contextual disparities in guidelines departures: Courtroom social contexts, guidelines compliance, and extralegal disparities", en: Criminology (Vol. 43, № 3), pp. 761-796.

KAHNEMAN, DANIEL Y TVERSKY, AmOS (1979): “Prospect theory: An analysis of decision under risk”, en: Econometrica (Vol. 47), pp. 263-291.

Kellough, GAIL y WORTLEY, SCOt (2002): "Remand for plea: Bail decisions and plea bargaining as commensurate decision", en: British Journal of Criminology (Vol. 42, № 1), pp. 186-210.

KRAMER, GReg M.; WolbranSky, MelindA y HeILBRUn, KIRK (2007): “Plea bargaining recommendations by criminal defense attorneys: Evidence strength, potential sentence, and defendant preference", en: Behavioral Sciences \& the Law (Vol. 25, № 4), pp. 575-585.

KRAMER, JOHN Y ULMER, JEFFREY T. (2002): “Downward departures for serious violent offenders: Local court 'corrections' to Pennsylvania's Sentencing Guidelines", en: Criminology (Vol 40, № 4), pp. 601-636.

LAMONTAGNE, LAUREL (2013): “Children under pressure: the problem of juvenile false confession and potential solutions", en: Western State University Law Review (Vol. 41, № 1), pp. 29-56.

LANDES, WILLIAM M. (1971): "An economic analysis of the courts", en: The Journal of Law and Economics (Vol. 14, № 1), pp. 61-107.

LANGeR, MAXIMo (2004): "From Legal Transplants to Legal Translations: The Globalization of Plea Bargaining and the Americanization Thesis in Criminal Procedure", en: Harvard International Law Journay (Vol. 45, № 1), pp. 1-64.

LAPP, KEVIN (2017): “Taking back juvenile confessions”, en: UCLA Law Review (Vol. 64), pp. 903-966.

LASCURAÍN SÁNCHEZ, JUAN ANTONIO Y GASCóN INCHAUSTI, FERNANDO (2018): “¿Por qué se conforman los inocentes?", en: InDret, Revista para el Análisis del Derecho (Vol. 3), pp. 1-28.

LeVERICK, FIONA (2004): "Tensions and Balances, Costs and Rewards: The Sentence Discount in Scotland", en: Edinburgh Law Review (Vol. 8, № 3), pp. 360-388.

MALloy, LindSAY C.; SHULman, ElizABeth P. y CAUfFMAN, ElizABETH (2014): “Interrogations, confessions, and guilty pleas among serious adolescent offenders", en: Law and Human Behavior (Vol. 38, № 29), pp. 181-193. 
MNOOKIN, ROBERT H. Y KORNHAUSER, LEWIS (1979): "Bargaining in the shadow of the law: The case of divorce", en: The Yale Law Journal (Vol. 88, № 5), pp. 950-997.

NieVA FenOLL, JORDI (2019): Derecho Procesal. Proceso penal (Valencia, Tirant lo Blanch), tomo III.

O'HEAR, MichaEL M. (2008): "Plea bargaining and procedural justice”, en: Georgia Law Review (Vol. 42), pp. 407-469.

Peterson-Badalı, Michele; Care, Stephanie y Broeking, Julia (2007): "Young People's Perceptions and Experiences of the Lawyer-Client Relationship", en: Canadian Journal of Criminology and Criminal Justice (Vol. 49, № 3), pp. 375-401.

PIZzI, WILLIAM T. (1996): "Fact-bargaining: An American phenomenon", en: Federal Sentencing Reporter (Vol. 8, № 6), pp. 336-338.

REDLiCH, Aluison D. (2010): "The susceptibility of juveniles to false confessions and false guilty pleas", en: Rutgers Law Review (Vol. 62), pp. 943-957.

Redich, Allison D.; SUmmers, Alicia y Hoover, Steven (2010): "Self-reported false confessions and false guilty pleas among offenders with mental illness", en: Law and Human Behavior (Vol. 34, № 1), pp. 79-90.

RedLiCH, Allison D. y BonVentre, CATHERINE L. (2015): “Content and comprehensibility of juvenile and adult tender-of-plea forms: Implications for knowing, intelligent, and voluntary guilty pleas", en: Law and Human Behavior (Vol. 39, № 2), pp. 162-176.

REDLICH, ALLISON D. (2016): "The validity of pleading guilty", en: Advances in Psychology and Law (Springer International Publishing, Charm) (Vol. 2, № 1), pp. 1-26.

Redich, Allison D. y SUMmers, Alicia (2012): "Voluntary, knowing, and intelligent pleas: Understanding the plea inquiry", en: Psychology, Public Policy and Law (Vol. 18, № 4), pp. 626-643.

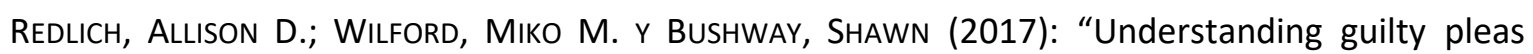
through the lens of social science", en: Psychology, Public Policy, and Law (Vol. 23, № 4), pp. 458471.

Redlich, Allison D.; Bibas, Stephanos; Edkins, VAnessa A. y MAdon, Stephanie (2017): “The psychology of defendant plea decision making", en: American Psychologist (Vol. 72, № 4), pp. 339-352.

RoBerTS, JENNy (2009): "Ignorance is effectively bliss: Collateral consequences, silence, and misinformation in the guilty-plea process", en: lowa Law Review (Vol. 95), pp. 119-194.

RODRÍGUEZ-ARIAS, ANTONIO MATEOS (2019): "Algunas reflexiones críticas sobre la conformidad en el proceso penal”, en: Anuario de la Facultad de Derecho (Vol. 35), pp. 167-194.

SAVITSKY, Douglas (2012): "Is Plea Bargaining a Rational Choice? Plea Bargaining as an Engine of Racial Stratification and Overcrowding in the United States Prison System", en: Rationality and Society (Vol. 24, № 2), pp. 131-167.

SCHNeIDeR, Ryan (2018): How Big is Too Big? The Potentially Coercive Effects of Plea Discount on Innocent Defendants (Theses, Dissertations and Culminating Projects).

SCHULHOFER, STEPHEN J. (1988): “Criminal justice discretion as a regulatory system", en: The Journal of Legal Studies (Vol. 17, № 1), pp. 43-82.

SCHULHOFER, STEPHEN J. (1992): “Plea bargaining as disaster", en: The Yale Law Journal (Vol. 101, № 8), pp. 1979-2009.

SCOTT, ROBERT E. Y STUNTZ, WILLIAM J. (1992): “Plea bargaining as contract”, en: The Yale Law Journal (Vol. 101, № 8), pp. 1909-1968.

Stancu, OANa y Varona Gómez, Daniel (2017): “¿Punitivismo también judicial? Un estudio a partir de las condenas penales por homicidio en España (2000-2013)", en: Revista Electrónica de Ciencia 
Penal y Criminología (№ 19-12), pp. 1-33. Disponible en: http://criminet.ugr.es/recpc/19/recpc1912.pdf [visitado el 12 de febrero de 2020].

StefFensmeier, Darrell Y Demuth, Stephen (2000): "Ethnicity and sentencing outcomes in U.S. federal courts: Who is punished more harshly?", en: American Sociological Review (Vol. 65), pp. 705-729.

Steffensmeier, Darrell; Kramer, John y Streifel, Cathy (1993): "Gender and imprisonment decisions", en: Criminology (Vol. 31, № 3), pp. 411-446.

STEFFENSMEIER, DARRELL; ULMER, JefFREY y KRAMER, JOHN (1998): "The interaction of race, gender, and age in criminal sentencing: The punishment cost of being young, black, and male", en: Criminology (Vol. 36, № 4), pp. 763-798.

Stephen, Frank. H.; Fazio, Giorgio y TAta, Cyrus (2008): "Incentives, criminal defence lawyers and plea bargaining", en: International Review of Law and Economics (Vol. 28, № 3), pp. 212-219.

TATA, CYRUS Y GORMLEY, JAY M. (2016): "Sentencing and plea bargaining: Guilty pleas versus trial verdicts", en: Criminal Courts and Prosecutors (Criminology \& Criminal Justice, Oxford University Press) (Vol. 1, № 1), pp. 1-29.

ToR, AVISHAlom; GazAl-Ayal, Oren y Garcia, Stephen M. (2010): “Fairness and willingness to accept plea bargain offers", en: Journal of Empirical Legal Studies (Vol. 7, № 1), pp. 97-116.

TYLeR, TOM R. (1990): Why People Obey the Law (New Haven, Yale University Press).

TYLER, TOM R. (2006): "Restorative justice and procedural justice: Dealing with rule breaking", en: Journal of social issues (Vol. 62, № 2), pp. 307-326.

Ulmer, JefFrey y BRADLEY, Mindy (2006): "Variation in trial penalties among serious violent offenders", en: Criminology (Vol. 44, № 3), pp. 631-670.

VIANO, EMILIO C. (2012): "Plea bargaining in the United States: A perversion of justice", en: Revue internationale de droit penal (Vol. 83, № 1), pp. 109-145.

VILOEN, JodI. L.; KLAVER, Jessica y ROESCH, RONALD (2005): “Legal decisions of preadolescent and adolescent defendants: Predictors of confessions, pleas, communication with attorneys, and appeals", en: Law and Human Behavior (Vol. 29, № 3), pp. 253-277.

Zarzalejos Nieto, Jesús (2011): "La conformidad de la persona jurídica imputada", en: Responsabilidad penal de las personas jurídicas. Aspectos sustantivos y procesales (Madrid, LaLey) (Vol. 1, № 1), pp. 271-285. 